\title{
How Does Market Network Structure Affect Participants' Benefits?
}

\author{
Yongli $\mathrm{Li}^{1,2}$ and Chong $\mathrm{Wu}^{1}$ \\ ${ }^{1}$ School of Management, Harbin Institute of Technology, Harbin 150001, China \\ ${ }^{2}$ Dipartimento di Economia Politica e Statistica, Università di Siena, 53100 Siena, Italy \\ Correspondence should be addressed to Chong Wu; wuc_hit@126.com
}

Received 25 January 2014; Accepted 16 April 2014; Published 19 May 2014

Academic Editor: Chien-Yu Lu

Copyright (c) $2014 \mathrm{Y}$. Li and C. Wu. This is an open access article distributed under the Creative Commons Attribution License, which permits unrestricted use, distribution, and reproduction in any medium, provided the original work is properly cited.

\begin{abstract}
A mathematical model is made to answer the question of how market network structure affects market participants' benefits. In this paper, one monopoly, one kind of goods, and $n$ consumers are assumed to form the market. The market network structure is defined as the mutual influences among consumers on purchase behaviors, and the benefits are defined as the sum of each consumer's utility as well as the profit of the monopoly. By completely knowing or not knowing the market network structure, the monopoly is designed to make decisions on price in such two cases. As a result, this paper finds that (1) when the market network structure is symmetric, completely knowing or not knowing the market structure network structure does not change the participants' benefits; (2) when the market network structure is asymmetric, knowing the network structure can help to improve the monopoly's profit, and meanwhile the consumers' benefits are identical on average in the statistical sense; (3) the increasing degree of the network structure matrix's asymmetry leads to the increasing benefits of the monopoly with fixing its original structure. Besides, policy implications and further work are also discussed.
\end{abstract}

\section{Introduction}

Complex networks form the backbone of social and economic life; accordingly many research papers have been published to analyze the complex network and its applications $[1,2]$. One thing of complex networks that attracted particular attention is that how its structure decides or affects its functions. As one interesting topic in the field of complexity, many scholars have explored the question from different angles; for example, Kauffman (1993) [3] utilized the theory of self-organization to establish a new model and provide the concept of landscape which lay a valid foundation for further research on organization structure and organization performance, and Solow et al. followed the concept of landscape to make a series of studies on the team structure and team performance based on mathematical models and simulation analysis [4-7]. Apart from the above example in the field of management science, the problem concerning the complex network structure and its corresponding function is also studied in the field of physics [8], knowledge-based systems [9], mathematics [10], and so on. In this paper, we set our model and analysis in the context of goods market, where we can easily define the market participants' benefits; accordingly the context facilitates us to explore the rule that how market network structure affects participants' benefits, namely, the embodiment of "structure and function" question in the field of economics.

This paper designs a goods market with one monopoly and many consumers. The relationships between these consumers likely formed based on friends, collaborators, competitors, or other sources [11-13] make up the market network. These consumers, as the network nodes, decide whether and how much to consume the goods according to the other consumers' responses. And the monopoly has the right to set different prices for different consumers based on his or her knowledge of the market network structure. This paper characterizes how much a monopoly can change his or her benefits by completely knowing or not knowing the market network structure when making the price decision and the resulting impact on consumers' benefits. 
Only few published literatures focused on the relationship between market network structure and market participants' benefits. There are some papers discussing the relationship between network structure and knowledge transmission such as Canessa and Riolo [14], Lazer and Friedman [15], and Hanaki et al. [16]. Also, there are also some papers exploring the network structure asymmetric and its impact on these consumers, such as Busse, et al. [17], Hortaçsu and Kastl [18], and Bhargava and Chen [19]. Although these cited papers are different from ours in terms of the research question, the methods adopted in the above papers can be helpful to ours. Our paper utilizes the techniques such as the mathematical model and the simulation analysis which are also adopted in the above papers. Thus, the results of this paper can be validated again and again accordingly the result would be robust.

The main work and contributions of this paper lies in two aspects: (1) we apply a utility function of considering the mutual influences among these consumers in the mathematical model, and the strength and structure of mutual influences are defined as the network structure in this paper; (2) we compare the differences of two kinds' market participants' benefits in two cases of knowing and unknowing the network structure through strict mathematical analysis and simulation analysis. Thus, based on the above discoveries, we find the rules that how market network structure affects monopoly's and consumers' benefits, respectively.

This paper is organized accordingly as follows. Section 2 presents the mathematical model and gives the definitions of network structure and benefits. Section 3 solves the model and obtains the optimal consumption level, pricing, and benefits. Section 4 compares the benefits in the two cases of knowing and unknowing the network structure and obtains three properties about the relationship between network structure and benefits. Section 5 concludes and discusses the future work.

\section{Model}

In this mathematical model, the market consists of a monopoly and a set of consumers denoted by $S=\{1,2, \ldots, n\}$. The monopoly produces one of the goods and has the rights to offer different prices to different consumers. In particular, $p_{i}$ is denoted as the price offered to consumer $i$ for one unit of goods. Then, the consumers decide the amount of the goods they will purchase at the announced price, and one consumer's decision is influenced by the others' purchases in the market. To describe the strength of the influence among these consumers, the matrix $\mathbf{A}$ is defined in Definition 1.

Definition 1 (the matrix $\mathbf{A}$ and network structure). The matrix $\mathbf{A}$ is defined as the strength matrix of the influence among these consumers, and $a_{i j}$, the $i j$ th entry of $\mathbf{A}$, represents the strength of the influence of consumer $j$ on $i$. Besides, the matrix $\mathbf{A}$ is also the definition of network structure discussed in this paper.

It is noted that we normalize $a_{i i}=0$ for all $i \in S$, since we focus on the influence from the others. Then, if $a_{i j}>0$, the influence of consumer $j$ on $i$ is positive, if $a_{i j}<0$, the influence of consumer $j$ on $i$ is negative, and if $a_{i j}=0$, the influence of consumer $j$ on $i$ does not exist. Furthermore, the above definition allows that $a_{i j} \neq a_{j i}$ which means that the strength of the influence of consumer $j$ on $i$ does not equal to that of consumer $j$ on $i$. So, the matrix A may not be symmetric.

Having the network structure of the market, we can further express each consumer's utility function in the following form (here, taking consumer $i$, e.g.):

$$
U_{i}\left(v_{i}\right)=\ln \left(1+b_{i} v_{i}-p_{i} v_{i}+v_{i} \sum_{j=1}^{n} a_{i j} v_{j}-c_{i} v_{i}^{2}\right),
$$

where $v_{i}$ is the amount of the goods that consumer $i$ decides to purchase and $v_{i} \geq 0$. To make the above utility function logical and reasonable, we provide the range of parameters as Assumption 2.

Assumption 2. For all $i \in S, p_{i}>0, c_{i}>0, b_{i}>0$, and $b_{i}>$ $p_{i}$.

It is natural that the price $p_{i}$, representing the cost of usage, is positive, because we need money to purchase goods in a market. The assumptions $b_{i}>0$ and $b_{i}>p_{i}$ imply that the corresponding goods are needed in the market and consuming it can increase the consumer's utility; besides, $c_{i}>$ 0 means that if the goods is consumed unduly, the utility will fall rapidly. According to Assumption 2, the sketch of $U_{i}\left(v_{i}\right)$ is shown in Figure 1. From Figure 1, we can find that when $v_{i}=0$, the corresponding $U_{i}\left(v_{i}\right)=0$. The above property is reasonable for representing the consumer's utility. Besides, with the increase of $v_{i}$, the $U_{i}\left(v_{i}\right)$, a unimodal and concave function, increases first and then decreases.

In the following, the monopoly aims to pursue the profit maximization, so he or she faces the problem shown as follows:

$$
\max _{p_{i}} \sum_{i=1}^{n}\left(p_{i} v_{i}-d v_{i}\right)
$$

from which we can find that the monopoly's core task is to price the goods for each consumer. In formula (2), $d$ denotes the cost of producing one unit of the goods. Formulas (1) and (2) are also related with the definition of benefits in this paper.

Definition 3 (benefits). All the consumers' benefits (denoted by $B e C$ ) are defined as the sum of each consumer's utility, and the monopoly's benefit (denoted by $B e M$ ) equals his profit which can be calculated from formula (2).

Solving formula (1) by maximizing the customers' utilities can obtain the equation of optimal consumption level, in which $p_{i}$ is exogenous and the matrixes $\mathbf{A}, c_{i}$, and $b_{i}$ are given. Taking the equation of optimal consumption level as constraint conditions of formula (2), we can obtain the optimal pricing and consequently achieve the expression of the monopoly's profit. The two steps are fundamental to solving the model, and the detailed processes appear in 


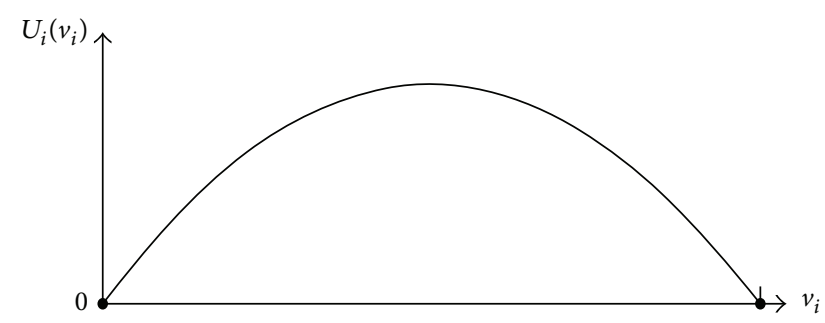

FIGURE 1: The sketch of $U_{i}\left(v_{i}\right)$.

Section 3 of this paper where the two cases of knowing the market network structure and not knowing will be analyzed.

What makes the mathematical model special and interesting lies in two folds: (1) its utility function contains the mutual influence among the consumers; (2) its solution procedure consists of two steps in which the effect of network structure (showed by the matrix $\mathbf{A}$ ) is easy to be uncovered and presented.

\section{Optimal Consumption Level, Discriminatory Pricing, and Benefits}

Optimal consumption level can be obtained by maximizing each consumer's utility expressed by formula (1); that is,

$$
\frac{\partial U_{i}\left(v_{i}\right)}{\partial v_{i}}=0, \quad \forall i \in S .
$$

As a result, we get the optimal consumption level $v_{i}$ as follows:

$$
v_{i}=\frac{\left(b_{i}-p_{i}+\sum_{j=1}^{n} a_{i j} v_{j}\right)}{2 c_{i}} ;
$$

however, formula (4) does not guarantee $v_{i} \geq 0$, so we need further to add the constraint into formula (4). The optimal consumption level shown above determines these consumers' consumptions, but it may not be perceived by the monopoly. Thus, two cases exist when the monopoly prices the goods for each consumer as follows.

Case 1. Not knowing the network structure reflected by the matrix A.

In this case, the monopoly does not perceive the matrix A's existence, so $a_{i j}=0$ for all $i, j \in S$. Accordingly, formula (4) in this case is

$$
2 c_{i} v_{i}=b_{i}-p_{i},
$$

which is the constraint condition of maximizing the monopoly's profits. It is noted that the value of $v_{i}$ must be nonnegative according to Assumption 2. Then, the monopoly faces such optimal problem as

$$
\begin{array}{ll}
\max _{p_{i}} & \sum_{i=1}^{n}\left(p_{i} v_{i}-d v_{i}\right), \\
\text { s.t. } \quad 2 c_{i} v_{i}=b_{i}-p_{i} .
\end{array}
$$

The results of solving the optimal problem expressed by formula (6) are

$$
\mathbf{p}=\frac{\mathbf{b}+\mathbf{d}}{2},
$$

in which $\mathbf{p}$ and $\mathbf{b}$ are the vector of all $p_{i}$ and $b_{i}(i \in S)$, respectively. Meanwhile, $\mathbf{d}$ is the $n \times 1$ vector consisting of $d$. After calculating the values of $p_{i}$, we put them into formula (4) and can obtain the values of $v_{i}$ as follows:

$$
\mathbf{v}=(\mathbf{C}-\mathbf{A})^{-1}\left(\frac{\mathbf{b}-\mathbf{d}}{2}\right),
$$

in which $\mathbf{v}$ is the vector of all $v_{i}$ and $\mathbf{C}$ is a diagonal matrix whose diagonal is composed of $2 c_{i}$. Subsequently, we can further express the consumers' benefit $(B e C)$ and the monopoly's benefit ( $B e M)$ in the condition of Case 1 as below:

$$
\mathrm{BeC}_{1}=\sum_{i=1}^{n} U_{i}\left(v_{i}\right)
$$

which can be calculated by putting formulas (7) and (8) into (1), and

$$
B e M_{1}=\left(\frac{\mathbf{b}-\mathbf{d}}{2}\right)^{T}(\mathbf{C}-\mathbf{A})^{-1}\left(\frac{\mathbf{b}-\mathbf{d}}{2}\right),
$$

which can be deduced by putting formulas (7) and (8) into (2).

Case 2. Knowing the network structure reflected by the matrix A.

In this case, the monopoly can grasp the network structure contained in the matrix A. Thus, the corresponding optimal problem can be expressed as

$$
\begin{array}{ll}
\max _{p_{i}} & \sum_{i=1}^{n}\left(p_{i} v_{i}-d v_{i}\right), \\
\text { s.t. } \quad 2 c_{i} v_{i}=b_{i}-p_{i}+\sum_{j=1}^{n} a_{i j} v_{j}, \quad v_{i} \geq 0,
\end{array}
$$

which is different from formula (6) in the constraint condition due to the monopoly's different perception of network structure. Similar to the solution of the optimal problem expressed by formula (6), the result of the above problem shown by formula (11) is

$$
\mathbf{p}=\mathbf{b}-(\mathbf{C}-\mathbf{A})\left(\mathbf{C}-\frac{\mathbf{A}+\mathbf{A}^{T}}{2}\right)^{-1} \frac{\mathbf{b}-\mathbf{d}}{2} .
$$

Next, putting the above result into formula (4), we can obtain the optimal consumption level:

$$
\mathbf{v}=\left(\mathbf{C}-\frac{\mathbf{A}+\mathbf{A}^{T}}{2}\right)^{-1} \frac{\mathbf{b}-\mathbf{d}}{2} .
$$


Accordingly, we can further express two kinds of benefits like the process of Case 1 as follows:

$$
\begin{aligned}
\mathrm{BeC}_{2} & =\sum_{i=1}^{n} U_{i}\left(v_{i}\right), \\
\mathrm{BeM}_{2} & =\left(\frac{\mathbf{b}-\mathbf{d}}{2}\right)^{T}\left(\mathbf{C}-\frac{\mathbf{A}+\mathbf{A}^{T}}{2}\right)^{-1}\left(\frac{\mathbf{b}-\mathbf{d}}{2}\right) .
\end{aligned}
$$

Lastly, it is noted that $(\mathbf{C}-\mathbf{A})^{-1}$ appears in formula (8) where we directly assume that the matrix $\mathbf{C}-\mathbf{A}$ is invertible, so we further need to discuss the condition of its invertibility. Here, a sufficient condition is given below as Assumption 4.

Assumption 4. Let $2 c_{i}>\max \left(\sum_{i=1}^{n}\left|a_{i j}\right|, \sum_{j=1}^{n}\left|a_{i j}\right|\right)$ for all $i \in S$.

The above assumption implies that $\mathbf{C}-\mathbf{A}$ is a strictly diagonally dominant matrix; thus it is invertible. Besides, under Assumption 4 , it is easily found that $\mathbf{C}-\left(\mathbf{A}+\mathbf{A}^{T}\right) / 2$ is also strictly diagonally dominant, so these formulas (12), (13), and (15) are well defined.

\section{How Does Network Structure Affect Market Participants' Benefits?}

In Definition 1, the matrix $\mathbf{A}$ has been defined as the network structure; here let us discuss how the matrix $\mathbf{A}$ affects the benefits of two kinds of market participants: $\mathrm{BeC}_{1}$ and $\mathrm{BeC}_{2}$, as well as $B e M_{1}$ and $B e M_{2}$.

First, it is easy to find Property 1 listed below.

Property 1. If $\mathbf{A}=\mathbf{A}^{T}$, then $B e C_{1}=B e C_{2}$ and $B e M_{1}=B e M_{2}$.

Property 1 implies that if every two consumers' mutual influences are equivalent and equal, the benefits of two cases are the same. Then, the correctness of Property 1 can be proved since formula (7) equals formula (12) and formula (8) equals formula (13) on the condition of $\mathbf{A}=\mathbf{A}^{T}$.

Second, when $\mathbf{A} \neq \mathbf{A}^{T}$ which means unequal mutual influences exist between two customers at least in the set, we can deduce that $B e C_{1}<B e C_{2}$ and $B e M_{1}<B e M_{2}$ through strict mathematical proof or simulation analysis. Property 2 summarizes this situation as follows.

Property 2. If $\mathbf{A} \neq \mathbf{A}^{T}, \operatorname{prob}\left(B e C_{1}<B e C_{2}\right)=\operatorname{prob}\left(B e C_{1}>\right.$ $\left.B e C_{2}\right)$ and $B e M_{1}<B e M_{2}$.

The mathematical proof and simulation analysis can be found in Appendices A and B. The property indicates that when $\mathbf{A}$ is asymmetric, knowing the network structure of the matrix $\mathbf{A}$ helps to improve the benefits of the monopoly, and meanwhile the benefits of consumers are unchanged in the sense of probability. The property is important and useful, because it encourages the monopoly to mine the network structure of mutual influences among these consumers and it also inspires that some mechanisms are designed to uncover the network structure contained in the matrix $\mathbf{A}$ which reflects the structure and strength of consumers' mutual influences.
Third, it is natural to ask whether the greater the matrix A's degree of asymmetry is, the larger the value of $\mathrm{BeM}_{2}-$ $B e M_{1}$ is. To answer this question, the degree of asymmetry of the matrix $\mathbf{A}$ (denoted by $e(\mathbf{A})$ ) is calculated firstly:

$$
e(\mathbf{A})=\left\|\mathbf{A}-\mathbf{A}^{T}\right\|_{1}=\sum_{i=1}^{n} \sum_{i=1}^{n}\left|a_{i j}-a_{j i}\right| .
$$

Then, we make simulation analysis and obtain the graphs as below. It is noted that all elements of $\mathbf{b}$ are set equal, which can eliminate the effect from the individual difference so as to highlight the effect of the matrix A. The detailed network structure of simulation analysis can be found in Appendix C.

Figure 2 shows the results that how the matrix A's degree of asymmetry $(e(\mathbf{A}))$ affects the value of $B e M_{2}-B e M_{1}$ which reflects the difference between knowing and unknowing the network structure of matrix $\mathbf{A}$. When the structure of matrix $\mathbf{A}$ is randomly changed in the simulation analysis, the value of $B e M_{2}-B e M_{1}$ with a larger $e(\mathbf{A})$ may be lower than that with a smaller $e(\mathbf{A})$ as shown in Figure 2, but the whole trend, depicted by the red line in Figure 2, is that with the increase of $e(\mathbf{A})$, the value of $B e M_{2}-B e M_{1}$ accordingly increases on average in the statistical sense. Besides, throughout further analysis, we can find that even if two matrixes have the same degree of asymmetry, their structures may be different greatly. Thus, the above results tell us that the value of $B e M_{2}-B e M_{1}$ is decided not only by $e(\mathbf{A})$, but also by the matrix's structure. The above findings are summarized into Property 3.

Property 3. The difference between $B e M_{1}$ and $B e M_{2}$ not only is influenced by $e(\mathbf{A})$ but also would be affected by the network structure. As a result, the value of $B e M_{2}-B e M_{1}$ with a larger $e(\mathbf{A})$ may be lower than that with a smaller $e(\mathbf{A})$, but on average, a positive relationship exists between the two variables.

Since the structure of matrix $\mathbf{A}$, independent of $e(\mathbf{A})$, is a factor of affecting the value $B e M_{2}-B e M_{1}$, we now fix its structure and find the relationship between $B e M_{2}-B e M_{1}$ and $e(A)$ without the influence from the structure. Accordingly, we first randomly generate the matrix $\mathbf{A}^{\prime}$, and once it is generated, it is fixed. Let $\mathbf{A}=\alpha \mathbf{A}^{\prime}$, where $\alpha$ is a random variable between 0 and 1 . Subsequently, we obtain the $e(\mathbf{A})$ and the corresponding $B e M_{2}-B e M_{1}$ when $\alpha$ is assigned randomly. It is noted that the larger the $\alpha$ is, the greater the matrix A's degree of asymmetry is, because $e(\mathbf{A})$ is an increasing function of $\alpha$.

In Figure 3, we have generated five different $\mathbf{A}^{\prime}$ s, so the lines with different colors correspond to different $\mathbf{A}^{\prime}$ s. One consistent pattern can be discovered from Figure 3, which is the main content of Property 4 as follows.

Property 4. When the structure of matrix $\mathbf{A}$ is fixed, the greater the matrix A's degree of asymmetry is, the larger the value of $B e M_{2}-B e M_{1}$ is.

\section{Conclusions and Future Research}

This paper makes a mathematical model to analyze the relationship between network structure and two kinds of 


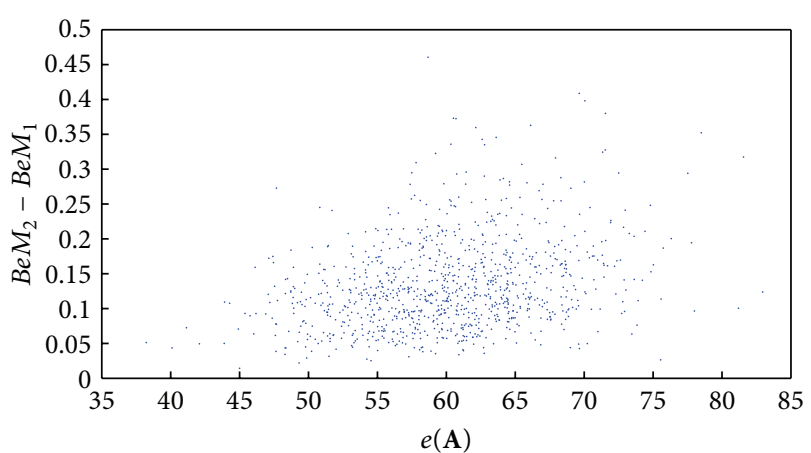

Figure 2: The relationship between $B e M_{2}-B e M_{1}$ and $e(\mathbf{A})$ when the structure of matrix $\mathbf{A}$ is free.

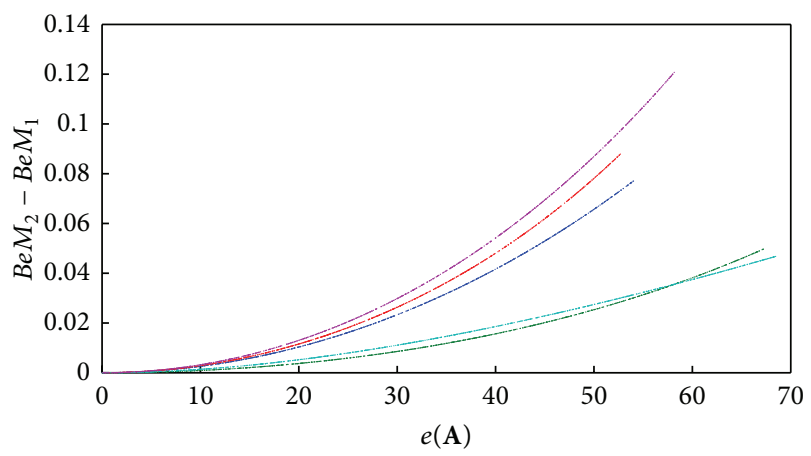

Figure 3: The relationship between $B e M_{2}-B e M_{1}$ and $e(\mathbf{A})$ when the structure of matrix $\mathbf{A}$ is fixed.

market participants' benefits. In the mathematical model, one monopoly, one kind of goods, and $n$ consumers are assumed to form the market, in which network structure is defined as the strength matrix of the influences among these consumers and benefits are defined as the sum of every consumer's utility as well as the profit of the monopoly. Subsequently, based on the maximization of the utility and the profit, the optimal consumption level, pricing, and benefits can be calculated so as to lay a foundation to compare the two cases of knowing the network structure and unknowing it. Throughout the comparison by strict mathematical proof and simulation analysis, we obtain three important results: (1) when the market network structure is symmetric, completely knowing or no knowing the market structure network structure does not change the two kinds participants' benefits; (2) when the market network structure is asymmetric, knowing the network structure can help to improve the monopoly's profit, and meanwhile the consumers' benefits are identical on average in the statistical sense; (3) the increasing degree of the network structure matrix's asymmetry leads to the increasing benefits of the monopoly with fixing its original structure. From the above three results, we suggest that the monopoly should mine the network structure of mutual influences among these consumers and some mechanisms should be designed to uncover the network structure because it is useful for lifting benefits.
However, this paper only points out that knowing or unknowing the network structure does not make change in the consumers' utilities in the sense of probability. In fact, some factors contained in the network structure matrix must take an effect to decide the consumers' utilities, since in some cases, knowing the network structure can increase the utilities and in others decreases them. To solve the above problem, it may need more detailed analysis on the structure of the network structure matrix and find in what conditions the utility can rise or drop. If so, the design of mechanism to uncover the network structure would be more meaningful because it could improve the consumers' utilities and the monopoly's profits at the same time.

\section{Appendices}

\section{A. Proof of Property 1: If $\mathbf{A} \neq \mathbf{A}^{T}$, then \\ $B e M_{1}<B e M_{2}$}

The above property aim to prove that when $\mathbf{A} \neq \mathbf{A}^{T}$, it holds that

$$
\begin{aligned}
& \left(\frac{\mathbf{b}-\mathbf{d}}{2}\right)^{T}(\mathbf{C}-\mathbf{A})^{-1}\left(\frac{\mathbf{b}-\mathbf{d}}{2}\right) \\
& \quad<\left(\frac{\mathbf{b}-\mathbf{d}}{2}\right)^{T}\left(\mathbf{C}-\frac{\mathbf{A}+\mathbf{A}^{T}}{2}\right)^{-1}\left(\frac{\mathbf{b}-\mathbf{d}}{2}\right) .
\end{aligned}
$$

Proof. Let $\mathbf{x}=(\mathbf{b}-\mathbf{d}) / 2$, the above formula equals

$$
\frac{\mathbf{x}^{T}(\mathbf{C}-\mathbf{A})^{-1} \mathbf{x}}{\mathbf{x}^{T}\left(\mathbf{C}-\left(\mathbf{A}+\mathbf{A}^{T}\right) / 2\right)^{-1} \mathbf{x}}<1 .
$$

To prove the above inequality holds, we need to mention that

$$
\begin{aligned}
& \frac{\mathbf{x}^{T}(\mathbf{C}-\mathbf{A})^{-1} \mathbf{x}}{\mathbf{x}^{T}\left(\mathbf{C}-\left(\mathbf{A}+\mathbf{A}^{T}\right) / 2\right)^{-1} \mathbf{x}} \\
& =\frac{\mathbf{x}^{T}\left[\left((\mathbf{C}-\mathbf{A})^{-1} / 2\right)+\left((\mathbf{C}-\mathbf{A})^{-T} / 2\right)\right] \mathbf{x}}{\mathbf{x}^{T}\left(\mathbf{C}-\left(\mathbf{A}+\mathbf{A}^{T}\right) / 2\right)^{-1} \mathbf{x}} \\
& \leq \max _{\|\mathbf{x}\|=1} \frac{\mathbf{x}^{T}\left[\left((\mathbf{C}-\mathbf{A})^{-1} / 2\right)+\left((\mathbf{C}-\mathbf{A})^{-T} / 2\right)\right] \mathbf{x}}{\mathbf{x}^{T}\left(\mathbf{C}-\left(\mathbf{A}+\mathbf{A}^{T}\right) / 2\right)^{-1} \mathbf{x}} .
\end{aligned}
$$

It is noted that $\left((\mathbf{C}-\mathbf{A})^{-1} / 2\right)+\left((\mathbf{C}-\mathbf{A})^{-T} / 2\right)$ and $\left(\mathbf{C}-\left(\mathbf{A}+\mathbf{A}^{T}\right) / 2\right)^{-1}$ are both symmetric matrix, so in order to prove the above inequality, we next need to prove that

$$
\frac{(\mathbf{C}-\mathbf{A})^{-1}}{2}+\frac{(\mathbf{C}-\mathbf{A})^{-T}}{2} \leq\left(\mathbf{C}-\frac{\mathbf{A}+\mathbf{A}^{T}}{2}\right)^{-1},
$$

according to the theorem of Rayleigh-Ritz, which is equivalent to

$$
\lambda_{\max }\left(\left[\frac{(\mathbf{C}-\mathbf{A})^{-1}}{2}+\frac{(\mathbf{C}-\mathbf{A})^{-T}}{2}\right] \cdot\left(\mathbf{C}-\frac{\mathbf{A}+\mathbf{A}^{T}}{2}\right)\right) \leq 1 .
$$


In fact,

$$
\begin{gathered}
{\left[\frac{(\mathbf{C}-\mathbf{A})^{-1}}{2}+\frac{(\mathbf{C}-\mathbf{A})^{-T}}{2}\right] \cdot\left(\mathbf{C}-\frac{\mathbf{A}+\mathbf{A}^{T}}{2}\right)} \\
=\frac{1}{2} \cdot \mathbf{I}+\frac{1}{4} \cdot\left((\mathbf{C}-\mathbf{A})^{-1}(\mathbf{C}+\mathbf{A})^{T}\right. \\
\left.+(\mathbf{C}-\mathbf{A})^{-T}(\mathbf{C}-\mathbf{A})\right) .
\end{gathered}
$$

Next, we have

$$
\begin{aligned}
& \lambda_{\max }\left(\frac{1}{2} \cdot \mathbf{I}+\frac{1}{4} \cdot\left((\mathbf{C}-\mathbf{A})^{-1}(\mathbf{C}+\mathbf{A})^{T}\right.\right.\left.\left.+(\mathbf{C}-\mathbf{A})^{-T}(\mathbf{C}-\mathbf{A})\right)\right) \\
& \leq \frac{1}{2}+\frac{1}{4} \cdot \lambda_{\max }\left((\mathbf{C}-\mathbf{A})^{-1}(\mathbf{C}+\mathbf{A})^{T}\right. \\
&\left.+(\mathbf{C}-\mathbf{A})^{-T}(\mathbf{C}-\mathbf{A})\right) .
\end{aligned}
$$
that

Thus, at this time, the whole problem becomes to prove

$$
\lambda_{\max }\left((\mathbf{C}-\mathbf{A})^{-1}(\mathbf{C}+\mathbf{A})^{T}+(\mathbf{C}-\mathbf{A})^{-T}(\mathbf{C}-\mathbf{A})\right) \leq 2 .
$$

To prove the above inequality, it is easy to find that

$$
\left|\lambda_{(\mathbf{C}-\mathbf{A})^{-1}(\mathbf{C}+\mathbf{A})^{T}}\right|=\left|\lambda_{(\mathbf{C}-\mathbf{A})^{-T}(\mathbf{C}-\mathbf{A})}\right|=1,
$$

and let $e^{i w}$ be the eigenvalue of $(\mathbf{C}-\mathbf{A})^{-1}(\mathbf{C}+\mathbf{A})^{T}$, where $w \in(0,2 \pi)$ (if and only if $\mathbf{A}=\mathbf{A}^{T}$, the eigenvalue of $(\mathbf{C}-\mathbf{A})^{-1}(\mathbf{C}+\mathbf{A})^{T}$ is a real number and $w=0$, so here $\left.w \neq 0\right)$. Then the eigenvalue of $(\mathbf{C}-\mathbf{A})^{-T}(\mathbf{C}-\mathbf{A})$ is $e^{-i w}$. Thus, we have

$$
\begin{aligned}
\lambda_{\max } & \left((\mathbf{C}-\mathbf{A})^{-1}(\mathbf{C}+\mathbf{A})^{T}+(\mathbf{C}-\mathbf{A})^{-T}(\mathbf{C}-\mathbf{A})\right) \\
& =e^{i w}+e^{-i w}=2 \cos w<2,
\end{aligned}
$$

when $w \in(0,2 \pi)$. Thus, the inequality has been proved.

Accordingly, if $\mathbf{A} \neq \mathbf{A}^{T}$, then $B e M_{1}<B e M_{2}$ holds.

\section{B. Validation of Property 1:}

$$
\operatorname{prob}\left(B e C_{1}<B e C_{2}\right)=\operatorname{prob}\left(B e C_{1}>B e C_{2}\right) \text {, When }
$$

$$
\mathbf{A} \neq \mathbf{A}^{T}
$$

Here, simulation analysis is used to validate the above result. The simulation is designed as follows: without loss of generality, let the elements of matrix $\mathbf{A}$ be assigned randomly values between -1 and $1 ; n=10, c_{i}=5, b_{i}=2$, and $d=1$, for all $i \in S$. By simulating 1000 times, we can obtain the graph showing the distribution of $\mathrm{BeC}_{2}-\mathrm{BeC}_{1}$ as shown in Figure 4 .

Figure 4 implies that the values of $\mathrm{BeC}_{2}-\mathrm{BeC}_{1}$ are symmetrically distributed about zero. So, we have validated that $\operatorname{prob}\left(B e C_{1}<B e C_{2}\right)=\operatorname{prob}\left(B e C_{1}>B e C_{2}\right)$ through simulation analysis.

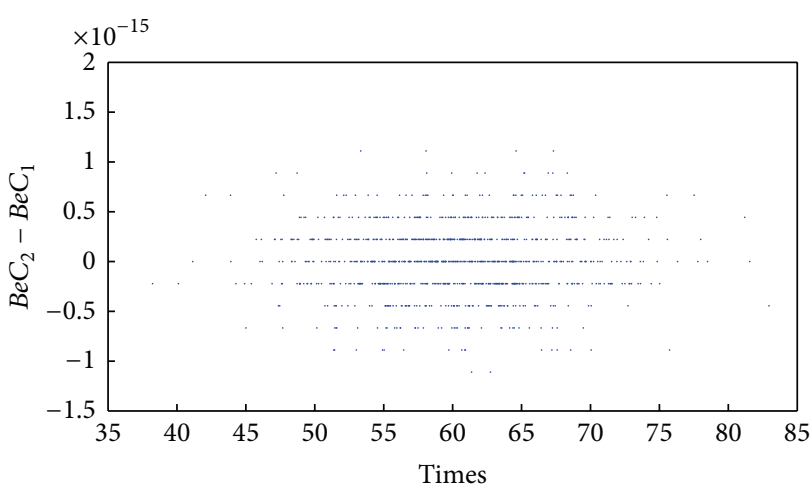

Figure 4: The distribution of $\mathrm{BeC}_{2}-\mathrm{BeC}_{1}$.

\section{The Detailed Network Structure of Simulation Analysis of Property 3}

The parameters here are identical with those in Appendix B, where the elements of matrix $\mathbf{A}$ are assigned randomly values between -1 and $1 ; n=10, c_{i}=5, b_{i}=2$, and $d=1$, for all $i \in S$.

\section{Conflict of Interests}

The authors declare that they have no conflict of interests regarding the publication of this paper.

\section{Acknowledgments}

This study is partly funded by National Natural Science Foundation of China (no. 71271070) and China Scholarship Council (no. 201306120159).

\section{References}

[1] H. Zhang, P. Zhao, J. Gao, and X.-M. Yao, "The analysis of the properties of bus network topology in Beijing basing on complex networks," Mathematical Problems in Engineering, vol. 2013, Article ID 694956, 6 pages, 2013.

[2] C.-Y. Chen and C.-W. Li, "A hybrid network model to extract key criteria and its application for brand equity evaluation," Mathematical Problems in Engineering, vol. 2012, Article ID 971303, 14 pages, 2012.

[3] S. A. Kauffman, The Origins of Order: Self-Organization and Selection in Evolution, Oxford University Press, New York, NY, USA, 1993.

[4] D. Solow and C. Leenawong, "Mathematical models for studying the value of cooperational leadership in team replacement," Computational \& Mathematical Organization Theory, vol. 9, no. 1, pp. 61-81, 2003.

[5] D. Solow and J. G. Szmerekovsky, "The role of leadership: what management science can give back to the study of complex systems," Emergence: Complexity \& Organization, vol. 8, no. 4, pp. 52-60, 2006.

[6] D. Solow and J. Szmerekovsky, "Mathematical models for explaining the emergence of specialization in performing tasks," Complexity, vol. 10, no. 1, pp. 37-48, 2004. 
[7] D. Solow, S. Piderit, A. Burnetas, and C. Leenawong, "Mathematical models for studying the value of motivational leadership in teams," Computational \& Mathematical Organization Theory, vol. 11, no. 1, pp. 5-36, 2005.

[8] A. W. Hübler, "Understanding complex systems: networks," Complexity, vol. 10, no. 3, p. 17, 2005.

[9] Y. Li, C. Wu, X. Wang, and S. Wu, "A tree-network model for mining short message services seed users and its empirical analysis," Knowledge-Based Systems, vol. 40, pp. 50-57, 2013.

[10] W. Lin and H.-F. Ma, "Failure of parameter identification based on adaptive synchronization techniques," Physical Review E, vol. 75, no. 6, Article ID 066212, 11 pages, 2007.

[11] S. Currarini, M. O. Jackson, and P. Pin, "Identifying the roles of race-based choice and chance in high school friendship network formation," Proceedings of the National Academy of Sciences of the United States of America, vol. 107, no. 11, pp. 4857-4861, 2010.

[12] M. Nermuth, G. Pasini, P. Pin, and S. Weidenholzer, "The network structureal divide," Games and Economic Behavior, vol. 78, pp. 21-30, 2013.

[13] Y. Li, C. Wu, P. Luo, and W. Zhang, "Exploring the characteristics of innovation adoption in social networks: structure, homophily, and strategy," Entropy, vol. 15, no. 7, pp. 2662-2678, 2013.

[14] E. Canessa and R. L. Riolo, "The effect of organizational communication media on organizational culture and performance: an agent-based simulation model," Computational \& Mathematical Organization Theory, vol. 9, no. 2, pp. 147-176, 2003.

[15] D. Lazer and A. Friedman, "The network structure of exploration and exploitation," Administrative Science Quarterly, vol. 52, no. 4, pp. 667-694, 2007.

[16] N. Hanaki, A. Peterhansl, P. S. Dodds, and D. J. Watts, "Cooperation in evolving social networks," Management Science, vol. 53, no. 7, pp. 1036-1050, 2007.

[17] M. Busse, J. Silva-Risso, and F. Zettelmeyer, “\$1,000 cash back: the pass-through of auto manufacturer promotions," American Economic Review, vol. 96, no. 4, pp. 1253-1270, 2006.

[18] A. Hortaçsu and J. Kastl, "Valuing dealers' informational advantage: a study of Canadian treasury auctions," Econometrica, vol. 80, no. 6, pp. 2511-2542, 2012.

[19] H. K. Bhargava and R. R. Chen, "The benefit of network structure asymmetry: when to sell to informed customers?" Decision Support Systems, vol. 53, no. 2, pp. 345-356, 2012. 


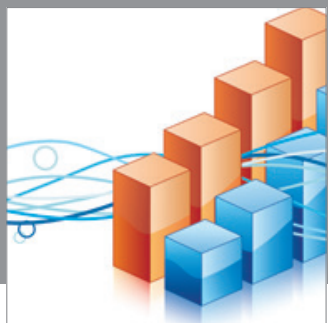

Advances in

Operations Research

mansans

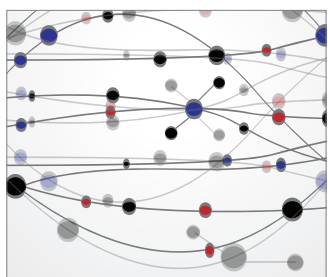

The Scientific World Journal
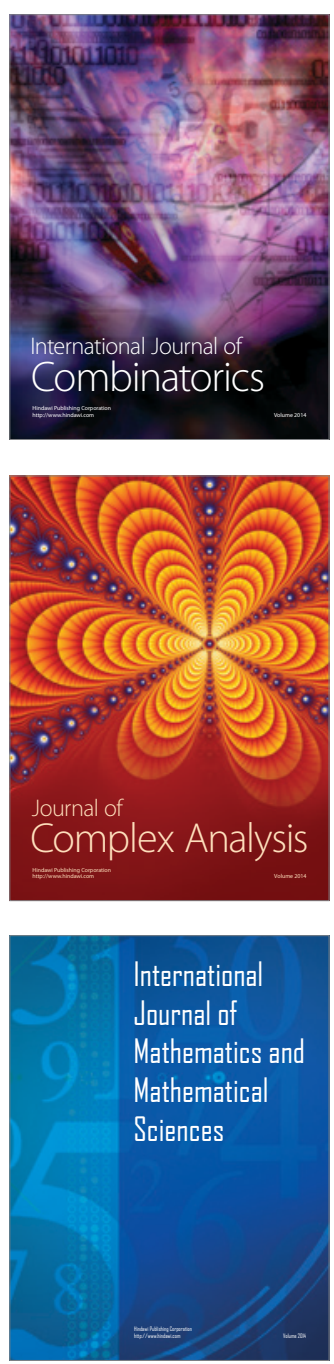
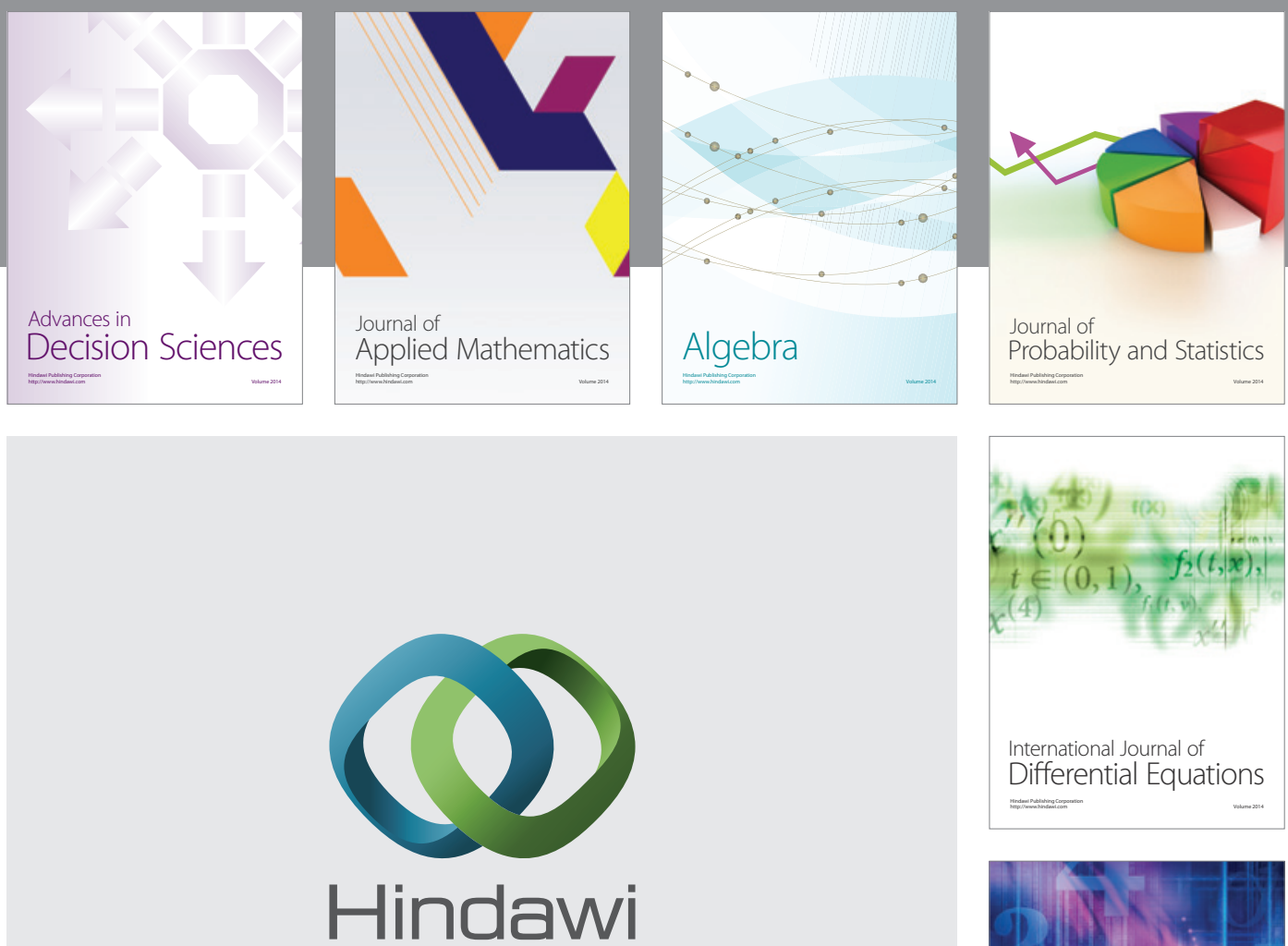

Submit your manuscripts at http://www.hindawi.com
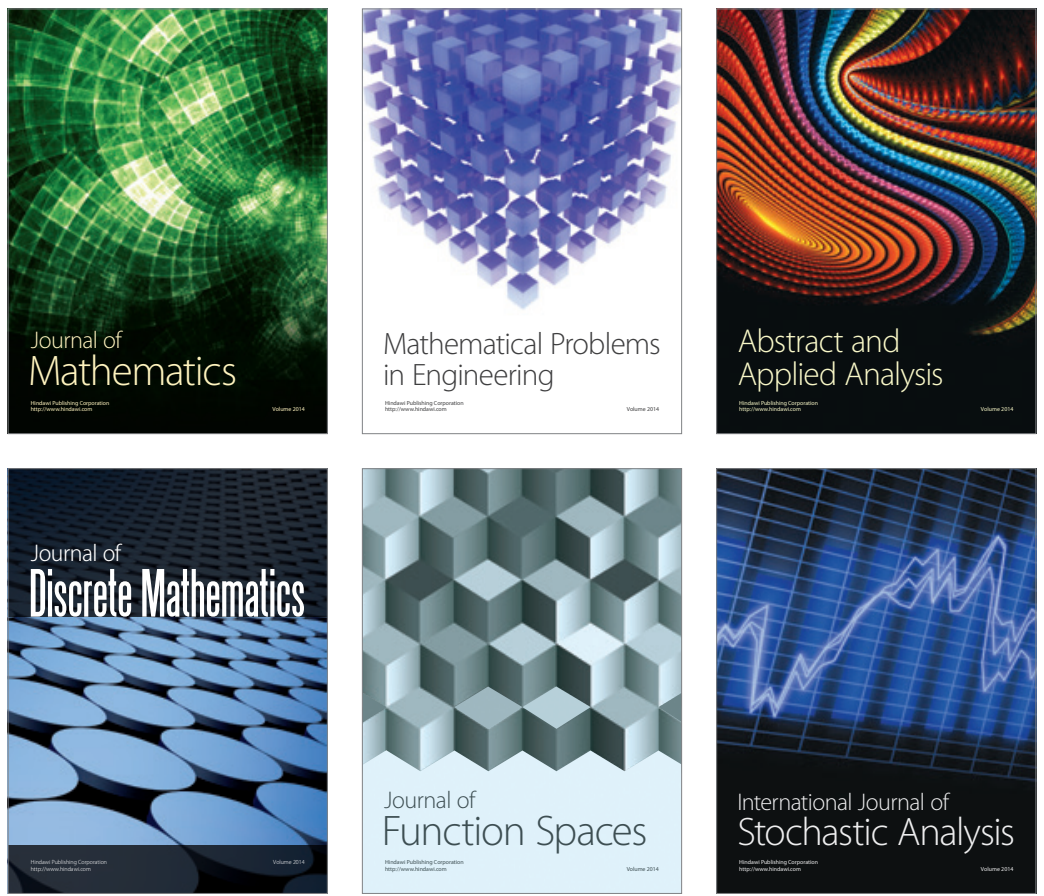

Journal of

Function Spaces

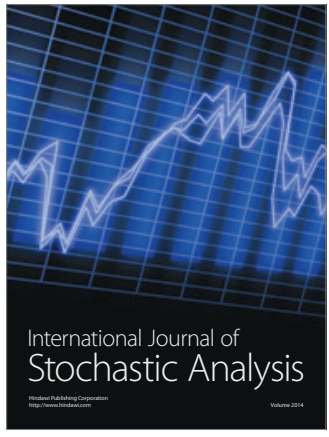

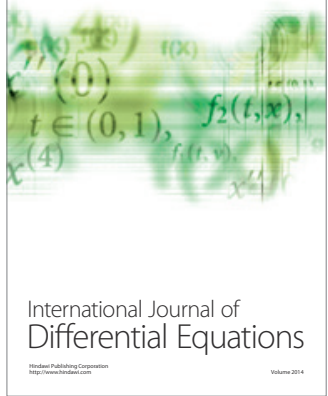
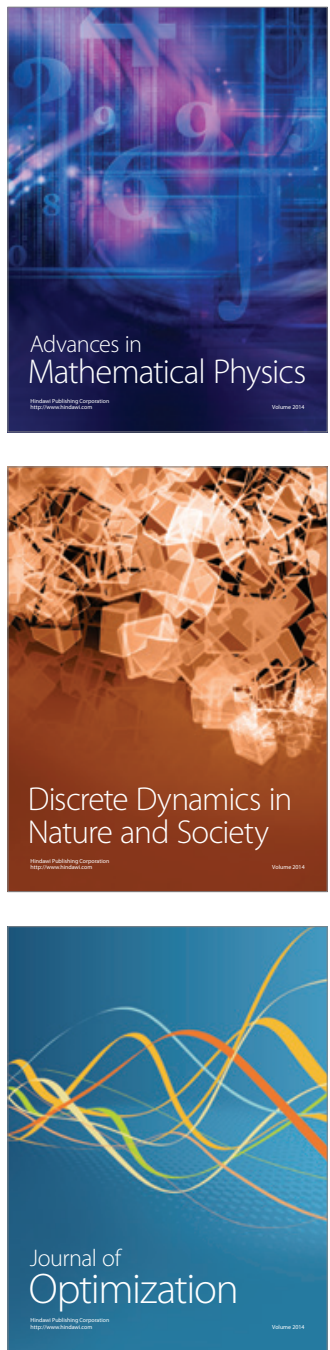